\title{
AGROMETEOROLOGIA
}

\section{ÍNDICE DE VEGETAÇÃO DO SENSOR MODIS NA ESTIMATIVA DA PRODUTIVIDADE AGRÍCOLA DA CANA-DE-AÇÚCAR $\left({ }^{1}\right)$}

\author{
MICHELLE CRISTINA ARAUJO PICOLI $\left({ }^{2}\right)$; BERNARDO FRIEDRICH THEODOR RUDORFF $\left({ }^{3 *}\right)$; \\ RODRIGO RIZZI $\left({ }^{4}\right)$; ANGÉLICA GIAROLLA $\left({ }^{5}\right)$
}

\begin{abstract}
RESUMO
A participação da cultura da cana-de-açúcar no fornecimento de matéria prima para produção de açúcar e também de álcool, como fonte alternativa de energia, tem sido relevante para o crescimento econômico do Brasil. Consequentemente, a disponibilidade de informações precisas sobre a produção agrícola dessa cultura é importante para auxiliar no planejamento e na tomada de decisões em toda a cadeia produtiva. O presente trabalho teve como objetivo estimar a produtividade agrícola de talhões de cana-de-açúcar para as safras 2004/2005 e 2005/2006, a partir de um modelo agronômico ajustado com dados orbitais. A inovação deste modelo consiste no uso do índice de área foliar (IAF) estimado a partir do produto índice de vegetação NDVI (Normalized Difference Vegetation Index) do sensor MODIS (Moderate Resolution Imaging Spectroradiometer) a bordo do satélite Terra da NASA (National Aeronautics Space Administration). O modelo agronômico explicou $31 \%$ e $25 \%$ da variação da produtividade observada entre talhões nos anos safra 2004/2005 e 2005/2006, respectivamente, o que se deve fundamentalmente ao uso das imagens NDVI do MODIS. O resultado do modelo pode ser usado para auxiliar e aprimorar a previsão da estimativa da produtividade feita in loco.
\end{abstract}

Palavras-chave: modelo agronômico, NDVI, sensoriamento remoto, imagens de satélite, sistema de informação geográfica.

$\left({ }^{1}\right)$ Recebido para publicação em 28 de abril de 2008 e aceito em 20 de março de 2009.

$\left({ }^{2}\right)$ Universidade Estadual de Campinas (UNICAMP), Faculdade de Engenharia Agrícola - FEAGRI, Caixa Postal 6011, 13083-875 Campinas (SP), Brasil. E-mail: michelle.picoli@feagri.unicamp.br

$\left({ }^{3}\right)$ Instituto Nacional de Pesquisas Espaciais (INPE), Divisão de Sensoriamento Remoto, Caixa Postal 515, 12210-970 São José dos Campos (SP), Brasil. E-mail: bernardo@ltid.inpe.br (*) Autor correspondente.

$\left({ }^{4}\right)$ Universidade Federal de Pelotas (UFPel), Faculdade de Agronomia Eliseu Maciel, Departamento de Engenharia Rural , 96010-900 Capão do Leão (RS), Brasil. E-mail: rodrigo.rizzi@ufpel.edu.br

${ }^{5}$ ) Instituto Nacional de Pesquisas Espaciais (INPE), Divisão de Satélites e Sistemas Ambientais Rodovia Presidente Dutra, km 40, 12630-000 Cachoeira Paulista (SP), Brasil. E-mail: angelica@cptec.inpe.br 


\title{
ABSTRACT \\ VEGETATION INDEX FROM MODIS SENSOR TO ESTIMATE SUGARCANE YIELD
}

\begin{abstract}
The contribution of sugarcane crop to provide raw material to produce sugar and also alcohol as an alternative energy source has been relevant to the economic growth of Brazil. Therefore, the availability of precise agricultural production information about this crop is important for planning and decisionmaking in the entire productive chain. The present work has the objective to estimate sugarcane yield in crop fields during the crop years 2004/2005 and 2005/2006, based on an agronomic model fit with orbital data. The innovation of this model consists in the use of the leaf area index (LAI) estimated from the NDVI (Normalized Difference Vegetation Index) produced by the MODIS sensor (Moderate Resolution Imaging Spectroradiometer) on board of the Terra satellite from NASA (National Aeronautics Space Administration). The agronomic model explained $31 \%$ and $25 \%$ of the yield variability among crop fields for the crop years 2004/2005 and 2005/2006, respectively, which is mainly attributed to use of NDVI images from MODIS. The model output should be useful to improve the precision of the crop yield estimation forecast performed in loco.
\end{abstract}

Key words: Agronomic model, NDVI, remote sensing, satellite images, Geographic Information System.

\section{INTRODUÇÃO}

A cana-de-açúcar é uma cultura de grande importância econômica para o Brasil, pois o país é o maior produtor de açúcar e etanol do mundo e ocupa posição de liderança na tecnologia de sua produção. São Paulo é o principal Estado produtor, responsável por quase $60 \%$ da cana processada no Brasil para produção de açúcar e álcool. Na safra 2007/2008, São Paulo produziu 295,5 milhões de toneladas de cana numa área de 3,50 milhões de ha (IBGE, 2008).

A produção de açúcar e de álcool depende, fundamentalmente, da quantidade de matéria prima disponível para moagem e que, por sua vez, depende da área cultivada, da produtividade agrícola e do açúcar total recuperável (ATR). Destes três fatores o mais complexo a ser estimado é a produtividade agrícola, pois está relacionada aos aspectos agronômicos da cultura e à variabilidade meteorológica, cujos efeitos sobre a produtividade são difíceis de serem quantificados.

Neste contexto, a estimativa da produtividade agrícola da cana-de-açúcar tem importância para o planejamento de uma série de atividades do agronegócio do setor sucroalcooleiro e também para o planejamento das operações nas usinas.

Em geral, as estimativas de produtividade agrícola das áreas canavieiras de uma usina são feitas antes do início da colheita por técnicos que percorrem os canaviais e observam o desenvolvimento das lavouras, atribuindo-lhes valores de produtividade com base na experiência adquirida e em informações de anos anteriores. Esta análise pode ser tendenciosa e não permite a análise dos erros envolvidos.
Estudos que utilizam dados meteorológicos e dados de sensoriamento remoto para a estimativa da produtividade de culturas agrícolas podem ser vistos em RudorfF (1985), RUdorfF e BATISTA (1990; 1991), RudorfF et al. (1995) e mais recentemente em FONTANA et al. (2001), BERKA et al. (2003) e Rizzi e Rudorff (2007). Estes estudos tiveram por base o emprego de uma variável: índice de vegetação, extraída de imagens de sensoriamento remoto, cuja informação está relacionada com o vigor vegetativo das culturas e, por conseguinte, à produtividade agrícola.

Entretanto, novos avanços são esperados no que se refere ao potencial do uso de imagens obtidas por satélites orbitais em modelos agronômicos para estimativa de produtividade de culturas agrícolas. Neste sentido, as imagens do sensor Moderate Resolution Imaging Spectroradiometer (MODIS) a bordo dos satélites Terra e Aqua (NASA/EOS), favorecem a aquisição de imagens livres de cobertura de nuvens e viabilizam o monitoramento da cultura ao longo de seu ciclo vegetativo e produtivo devido à sua resolução temporal de dezesseis dias.

Dentre os produtos gerados a partir dos dados coletados por este sensor, o índice de vegetação Normalized Difference Vegetation Index (NDVI) tem apresentado ampla aplicação em estudos ambientais (RUdORfF et al., 2007). As imagens do NDVI são fornecidas a cada 16 dias, com georreferenciamento, correção atmosférica e podem ser adquiridas em resolução espacial de $250 \times 250 \mathrm{~m}$. Até o presente momento, não se tem conhecimento em literatura de modelos que utilizaram dados do sensor MODIS para estimar a produtividade da cana-de-açúcar em escala de talhão. 
Este trabalho teve como objetivo estimar a produtividade em talhões de cana-de-açúcar nas safras 2004/2005 e 2005/2006 utilizando um modelo agronômico em um Sistema de Informação Geográfica (SIG), com base em dados de sensoriamento remoto e meteorológicos. As estimativas geradas por este modelo devem auxiliar os técnicos de usinas em suas estimativas de produtividade feita in loco.

\section{MATERIAL E MÉTODOS}

A área de estudo abrange as lavouras de canade-açúcar, localizadas no Centro-Norte do Estado de São Paulo, entre as coordenadas $20^{\circ} 30^{\prime}$ a $21^{\circ} 40^{\prime}$ de latitude Sul e $49^{\circ} 20^{\prime}$ a $48^{\circ} 30^{\prime}$ de longitude Oeste. Esta área envolve 21 municípios e representa uma extensão de aproximadamente $4.032 \mathrm{~km}^{2}$, sendo a área plantada com cana-de-açúcar equivalente a aproximadamente seis mil hectares. Os talhões selecionados foram os que se encontravam no estágio de cana soca $\left({ }^{6}\right)(\mathrm{CS})$, que representam mais de $80 \%$ da área cultivada, e que fossem maiores que um hectare.

As variáveis meteorológicas requeridas pelo modelo de estimativa de produtividade são: temperatura média do ar $\left({ }^{\circ} \mathrm{C}\right)$; velocidade do vento a $2 \mathrm{~m}$ de altura $\left(\mathrm{km} \mathrm{dia}^{-1}\right)$; umidade relativa do ar (\%); radiação solar $\left(\mathrm{W} \mathrm{m}^{-2}\right)$ e precipitação pluvial ( $\mathrm{mm} \mathrm{dia}^{-}$ ${ }^{1}$ ). Em função da dificuldade de acesso em tempo oportuno a dados provindos de estações meteorológicas e sua introdução em modelos desta natureza, optou-se por utilizar os dados oriundos do modelo de previsão de tempo ETA (BLACK, 1994), em operação no Centro de Previsão de Tempo e Estudos Climáticos (CPTEC/INPE). Os dados utilizados do modelo ETA são amostras pontuais com resolução horizontal de $40 \mathrm{~km}$. Estes dados foram organizados em médias quinzenais, com exceção da precipitação pluvial, que foi somada quinzenalmente, pois o modelo calculou a produtividade agrícola da cana-deaçúcar a cada quinze dias. Todos os cálculos envolvendo a utilização dos dados oriundos do modelo ETA e sua introdução no modelo agronômico estão descritos em Rizzi et al. (2006). A partir das amostras pontuais foram geradas grades regulares (matrizes) com resolução espacial de $250 \mathrm{~m}$, pelo método de interpolação vizinho mais próximo, para cada variável meteorológica, visto ser esta a resolução espacial do produto NDVI do sensor MODIS (produto MOD13Q1, coleção 4; LATORRE et al., 2007).

${ }^{6}$ ) Cana soca (CS) refere-se à rebrota da cana-de-açúcar após o primeiro corte na colheita.
Os dados utilizados no modelo referente à Capacidade de Água Disponível (CAD) dos solos foram fornecidos pela Usina Catanduva para cada um dos talhões de cana da área de estudo. O cálculo da CAD leva em consideração: o tipo de solo, a capacidade de campo, o ponto de murcha permanente, a densidade global do solo e a profundidade média de exploração das raízes das culturas agrícolas (CAmargo, 1971; Pereira et al., 1997).

Os dados de altitude (m), também utilizados no cálculo da estimativa de produtividade, sob a forma de um Modelo Digital de Elevação (MDE), com resolução espacial de $1 \times 1 \mathrm{~km}$, foram adquiridos junto ao Centro de Dados do EROS/USGS (Earth Resources Observation and Science/United States Geological Survey). Estes dados foram reamostrados para a resolução espacial de $250 \mathrm{~m}$ pelo mesmo motivo citado anteriormente.

O modelo agronômico utilizado neste trabalho foi inicialmente proposto por DoOREnBOS e KASSAM (1979) e está fundamentado no efeito da deficiência hídrica sobre a produtividade agrícola. A quantificação deste efeito se dá através do somatório da relação entre a evapotranspiração real e a potencial $(\mathrm{ER} / \mathrm{EP})$ e de um fator de resposta à produtividade (ky) que relaciona a deficiência hídrica com a demanda de água pela cultura nos diferentes estádios fenológicos para a formação da produtividade agrícola. Esse modelo foi escrito em linguagem FORTRAM por RUDORFF (1985) e mais tarde implementado por BERKA et al. (2003) em um Sistema de Informação Geográfica (SPRING - Sistema de Processamento de Informações Georeferenciadas), desenvolvido pelo Instituto Nacional de Pesquisas Espaciais (INPE). Com a possibilidade de se estimar o índice de área foliar (IAF) a partir de imagens de sensoriamento remoto o modelo passou a revelar aspectos relevantes para estudos em escalas regionais (Rizzi e RudorfF, 2007).

Para tentar representar no modelo a variabilidade espacial do vigor vegetativo dos talhões de cana, foram utilizadas imagens NDVI do sensor MODIS como variável espectral no modelo agronômico. Cada imagem NDVI do sensor MODIS foi primeiramente utilizada para estimar a fração da cobertura do solo $(\mathrm{Fc})$, adaptado do método proposto por CHOUDHURY et al. (1994), conforme a Equação 1:

$$
F c=1-\left(\frac{N D V I_{\text {max }}-N D V I}{N D V I_{\text {max }}-N D V I_{\text {min }}}\right)^{0,9}
$$

em que, NDVImax é o valor máximo do NDVI da imagem, NDVImin é o valor mínimo do NDVI da imagem e NDVI é o valor do pixel para o qual está sendo calculado o valor de Fc. 
O Índice de Área Foliar (IAF) foi estimado pela Equação 2, seguindo a metodologia sugerida por NORMAN et al. (2003).

$$
I A F=-2 \operatorname{Ln}(1-F c)
$$

O modelo agronômico utiliza o IAF para o cálculo da produtividade através do fator de compensação do crescimento (Fcc), que por sua vez independe do tipo da cultura, como indica a Equação 3, ajustada por BERKA et al. (2003), com base em DOORENBOS e KASSAM (1979):

$$
F C C=0,515-e^{\left(-0,667-\left(0,515^{*} I A F\right)\right.}
$$

sendo os valores de IAF obtidos a partir da Equação 2. Além dos dados já citados, alguns parâmetros específicos da cultura da cana-de-açúcar foram necessários para que o modelo agronômico fosse executado, tais como: o coeficiente de cultura (kc), o fator de resposta à produtividade $(\mathrm{ky})$ e os valores de profundidade do sistema radicular (D). Tais parâmetros tiveram seus valores ajustados de acordo com o desenvolvimento da planta para cada quinzena. Maiores informações sobre tais parâmetros podem ser encontradas em DoOREnbos e KASSAM (1979) e RUdORFF (1985).

O fator de produtividade agrícola (FPA), outro parâmetro do modelo, possui um elevado peso no cálculo da estimativa da produtividade e tem o mesmo valor para todo o ciclo da cultura, independentemente do ano safra. O modelo estima a produtividade com base na matéria seca total da planta, e o FPA é multiplicado pelo valor de produtividade obtido em cada quinzena. Neste trabalho, o valor do FPA utilizado foi 2,9, com base no trabalho de RUDORFF (1985). A soma das produtividades parciais em cada quinzena corresponde ao valor da produtividade final estimada. Após o ajuste dos parâmetros, foi realizado o cálculo da produtividade agrícola em escala de talhão, para o período de outubro a março. Neste período, ocorre o maior acúmulo de biomassa pela cultura conforme se verificou em uma avaliação prévia feita com nos valores de NDVI de todos os talhões, para as safras 2004/2005 e 2005/2006.

O coeficiente de determinação ( $R$ ) foi utilizado para avaliar o desempenho do modelo por meio da análise de regressão entre os valores de produtividade estimados pelo modelo agronômico e os valores de produtividade observados pela Usina. A quantificação dos erros sistemático (Es) e aleatório (Ea), e do índice de concordância " $\mathrm{d}$ " foi feito segundo Willmot et al. (1985). O "R" indica a precisão do modelo, ou seja, quanto a oscilação da variável dependente é explicada pelas variáveis independentes. O índice " $\mathrm{d}$ ", com variação entre 0 e 1 , indica o grau de exatidão entre os valores estimados e observados, quanto mais próximo de 1, melhor a exatidão do modelo em estimar a produtividade. Quanto mais próximos os dados estiverem da linha 1:1, melhor a correlação entre os valores estimados e os observados. A análise também fornece informações sobre o erro absoluto médio (EAM) e sobre o erro quadrático médio (EQM).

Adicionalmente, o modelo agronômico foi executado quinzenalmente utilizando apenas o valor médio de NDVI de todos os talhões, a fim de avaliar a influência da variável espectral (NDVI/MODIS) na variabilidade espacial das estimativas de produtividade.

Por fim, foi feita uma avaliação estatística, teste $t$ (SteEl e TORRIE, 1980), para comparar a média da produtividade estimada pelo modelo com a média da produtividade observada pela Usina. Esta avaliação permitiu analisar se existe diferença significativa entre os valores médios de produtividade estimados pelo modelo em relação aos valores médios da produtividade observada.

\section{RESULTADOS E DISCUSSÃO}

Os gráficos da figura 1 representam a variação temporal dos valores de NDVI dos talhões de cana nas safras 2004/2005 (A) e 2005/2006 (B). Considerando que os valores de NDVI estão relacionados com a quantidade de biomassa da cultura, é possível afirmar, a partir destes gráficos, que o período de maior acúmulo de biomassa pela cultura ocorre de outubro a março, concordando com o relatado por CASTRO (1999). Com base nesse resultado, o cálculo da estimativa da produtividade da cana pelo modelo agronômico foi feito para o período compreendido entre outubro de um ano e março do ano seguinte.

A avaliação da precisão da estimativa do modelo é apresentada na tabela 1. Observou-se que para a safra 2004/2005 o modelo agronômico explicou $31 \%$ da variabilidade da produtividade observada com erro quadrático médio (EQM) de $20,9 \mathrm{t} \mathrm{ha}^{-1}$. Para a safra 2005/2006 o modelo explicou 25\% da variabilidade da produtividade observada com EQM de 19,2 $\mathrm{t} \mathrm{ha}^{-1}$ (Figura 2). O índice de concordância (d) foi igual a 0,87 e 0,62 para as safras 2004/2005 e 2005/2006, respectivamente.

O valor médio da produtividade observada dos talhões foi de $91,9 \mathrm{t} \mathrm{ha}^{-1}$ e $79,2 \mathrm{t} \mathrm{ha}^{-1}$ para as safras 2004/2005 e 2005/2006 respectivamente. Portanto, o modelo agronômico subestimou a produtividade média em $14,6 \%\left(13,4 \mathrm{tha}^{-1}\right)$ na safra $2004 / 2005$ e superestimou em $1,8 \%\left(1,4 \mathrm{t} \mathrm{ha}^{-1}\right)$ na safra $2005 / 2006$. 

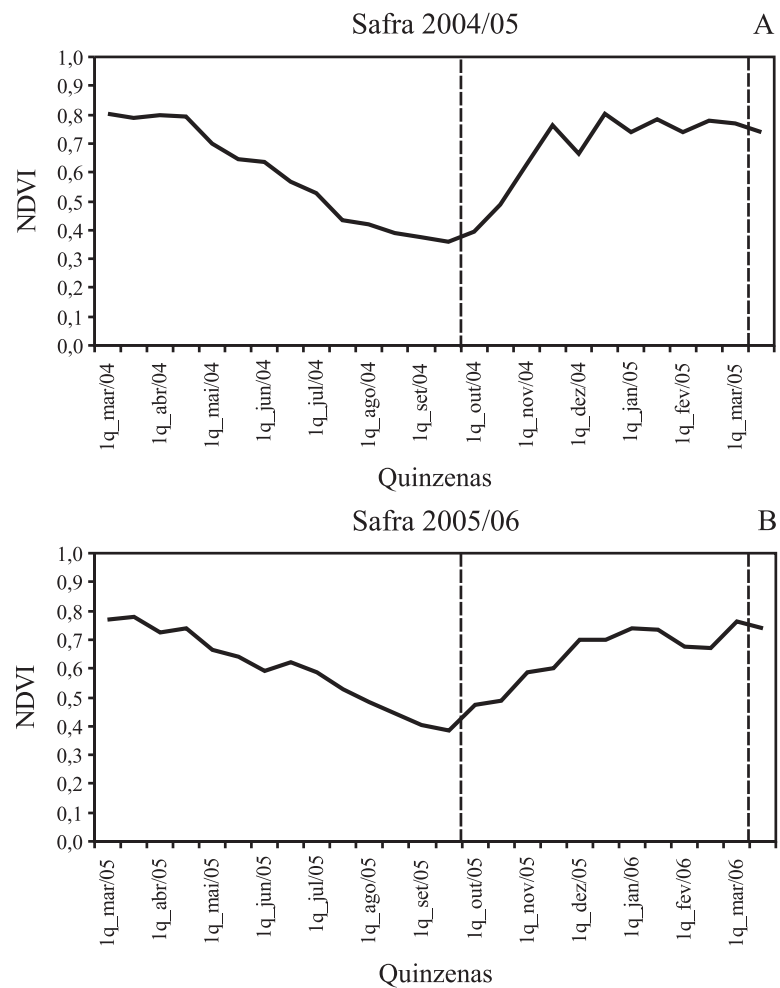

Figura 1. Variação temporal do NDVI (sensor MODIS) dos talhões nas safras (A) 2004/2005 e (B) 2005/06. As linhas tracejadas representam o início e o fim do período de maior acúmulo de biomassa da cultura (outubro a março).

Tabela 1. Produtividade média estimada pelo modelo agronômico (PME), produtividade média observada $(\mathrm{PMO})$, coeficiente de determinação $\left(\mathrm{R}^{2}\right)$, índice de concordância (d), erro absoluto médio (EAM), erro sistemático (ES), erro aleatório (EA) e erro quadrático médio (EQM) do modelo agronômico para as safras $2004 / 2005$ e $2005 / 2006$

\begin{tabular}{lcc}
\hline \multirow{2}{*}{ Parâmetro } & \multicolumn{2}{c}{ Safra } \\
\cline { 2 - 3 } & $2004 / 2005$ & $2005 / 06$ \\
\hline $\begin{array}{l}\text { Produtividade média } \\
\text { estimada (PME) }\end{array}$ & $78,5 \mathrm{t} \mathrm{ha}^{-1}$ & $80,6 \mathrm{t} \mathrm{ha}^{-1}$ \\
$\begin{array}{l}\text { Produtividade média } \\
\text { observada (PMO) }\end{array}$ & $91,9 \mathrm{t} \mathrm{ha}^{-1}$ & $79,2 \mathrm{t} \mathrm{ha}{ }^{-1}$ \\
Coeficiente de determinação $\left(\mathrm{R}^{2}\right)$ & 0,31 & 0,25 \\
$\begin{array}{l}\text { Índice de concordância (d) } \\
\text { Erro absoluto médio (EAM) }\end{array}$ & 0,87 & 0,62 \\
$\begin{array}{l}\text { Erro sistemático (ES) } \\
\text { Erro aleatório (EA) }\end{array}$ & $8,6 \mathrm{t} \mathrm{ha}^{-1}$ & $9,4 \mathrm{t} \mathrm{ha}^{-1}$ \\
Erro Quadrático Médio (EQM) & $20,9 \mathrm{t} \mathrm{ha}^{-1}$ & $19,2 \mathrm{t} \mathrm{ha}^{-1}$
\end{tabular}
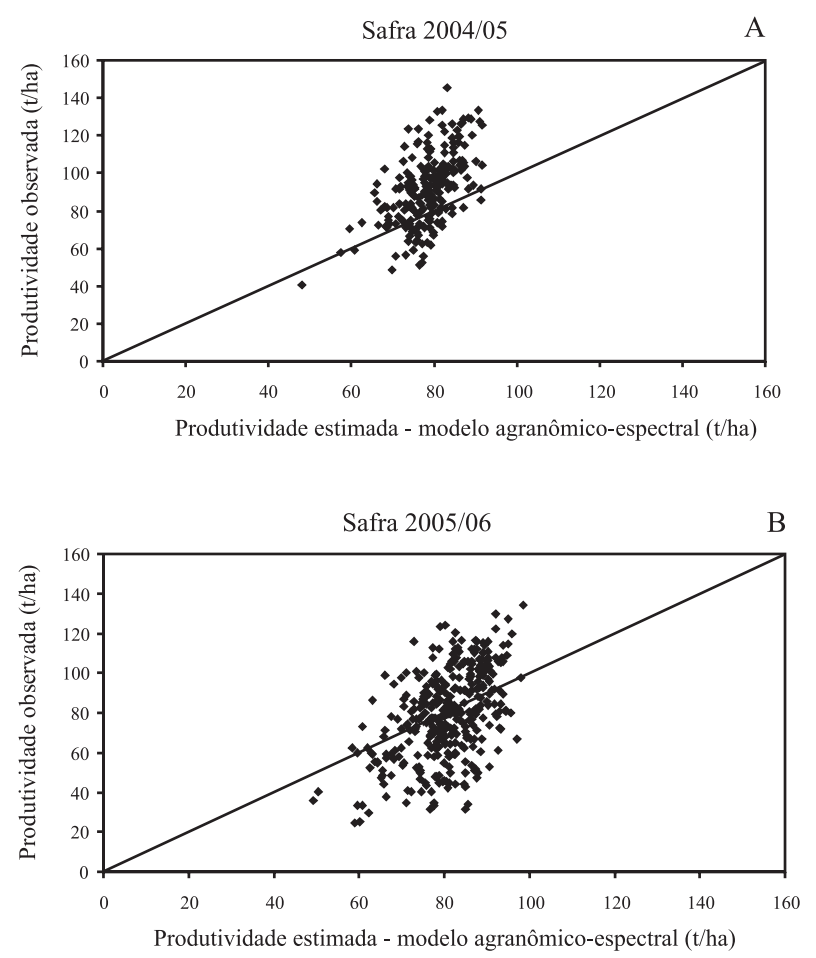

Figura 2. Relação entre a produtividade observada e a produtividade estimada pelo modelo agronômico nas safras (A) 2004/2005 e (B) 2005/2006.

O índice de concordância "d" próximo de "um" caracteriza concordância quase perfeita entre o dado estimado e o dado observado, portanto para a safra $2004 / 2005(d=0,87)$ o modelo conseguiu estimar a produtividade com mais exatidão do que para a safra 2005/2006 $(d=0,62)$. O teste $t$ para comparação de médias entre os valores de produtividade estimados pelo modelo agronômico e pela produtividade média observada na usina revelou que existe diferença significativa nas duas safras $(p=0,05)$.

Uma análise preliminar do resultado pode levar à conclusão de que o modelo agronômico não teve um desempenho satisfatório e que outros fatores (p. ex. pragas, doenças, adubação) não considerados exerceram influência significativa na formação da produtividade agrícola da cana. Outra hipótese é a de que a resolução temporal e/ou espacial das variáveis consideradas não seja adequada para representar seus efeitos sobre a produtividade da cana. As variáveis meteorológicas do modelo ETA são fornecidas em escala diária (embora tenham sido utilizados valores quinzenais no presente estudo), no entanto foram utilizadas em baixa resolução espacial $(40 \mathrm{~km})$ e isso pode ter dificultado a observação de variações entre os talhões de cana em uma mesma célula ou pixel. Por exemplo, a precipitação pluvial é a variável que exerce maior influência na produtividade agrícola e pode ter alta variabilidade espacial dentro de uma célula de $40 \mathrm{~km}$. 
De forma análoga, os pixels do NDVI com resolução espacial de $250 \mathrm{~m}(6,25 \mathrm{ha})$ podem não estar representando a variação média da resposta espectral de um talhão de cana (p. ex., talhão pequeno representado por uma janela menor que $3 \times 3$ pixels). A influência da resolução das variáveis do modelo necessita ser analisada em maior profundidade; entretanto, é preciso levar em conta as atuais limitações tecnológicas para obtenção de dados com melhores resoluções. Outra hipótese levantada neste trabalho é que a resolução espacial do modelo proposto por DoORenbos e KASSAM (1979) não é adequada para estimar a produtividade agrícola da cana-de-açúcar em escala de talhão, que para a área de estudo variavam de um à quarenta hectares, o que concordaria com estudo feito por TERAMOTO (2003).

Apesar disso, quando se utilizou a média do NDVI de todos os talhões de cana da área de estudo, com o intuito de verificar a influência dos valores espaciais do NDVI o resultado revelou que nestas condições o modelo não explica qualquer variação na produtividade observada (Figura 3). Esse fato sugere que o NDVI transformado em valores de IAF, é o grande responsável por explicar $31 \%$ e $25 \%$ da variação na produtividade nas safras 2004/2005 e 2005/2006, respectivamente (Figura 3). Indica, ainda, que a resolução espacial e/ou temporal de uma ou mais variáveis meteorológicas utilizadas não é adequada para explicar a variação da produtividade entre talhões de cana. Assim, é possível que a melhoria tanto na resolução espacial dos dados do modelo ETA quanto na resolução temporal do modelo agronômico possa levar a estimativas mais precisas da produtividade da cana. Esta suposição também é válida para a resolução espacial das imagens NDVI do sensor MODIS. RUDORFF e BATISTA (1990) utilizaram um índice de vegetação, semelhante ao NDVI, com resolução espacial de 80 metros, mas em apenas uma data em todo o ciclo anual da cultura. Neste caso, os autores utilizaram o índice vegetativo como variável independente, juntamente com a estimativa do modelo agronômico, para estimar a produtividade da cana por meio de regressão, resultando em um coeficiente de determinação $\left(R^{2}\right)$ que variou de 0,50 a 0,69 . Esse valor indica que uma melhora na resolução espacial das imagens traga grandes benefícios para o modelo de estimativa de produtividade. Porém, cabe salientar que as resoluções espacial e temporal de uma imagem de sensoriamento remoto não são independentes, ou seja, quando se aumenta a resolução temporal, invariavelmente se diminui a resolução espacial. Entretanto, com o avanço tecnológico observado nas duas últimas décadas, as resoluções das imagens têm melhorado de forma significativa permitindo que se disponha, atualmente, de um sensor como o MODIS, que adquire imagens com resolução temporal quase diária em uma resolução espacial de $250 \mathrm{~m}$ (6,25 ha), o que representa considerável ganho para melhorar a estimativa da produtividade de culturas agrícolas (Rizzi e RudorfF, 2007). Assim, com este trabalho constata-se que os dados de sensoriamento remoto podem vir auxiliar e complementar a informação coletada in loco pelos técnicos da Usina Catanduva para estimar com maior precisão a produtividade dos talhões de cana.
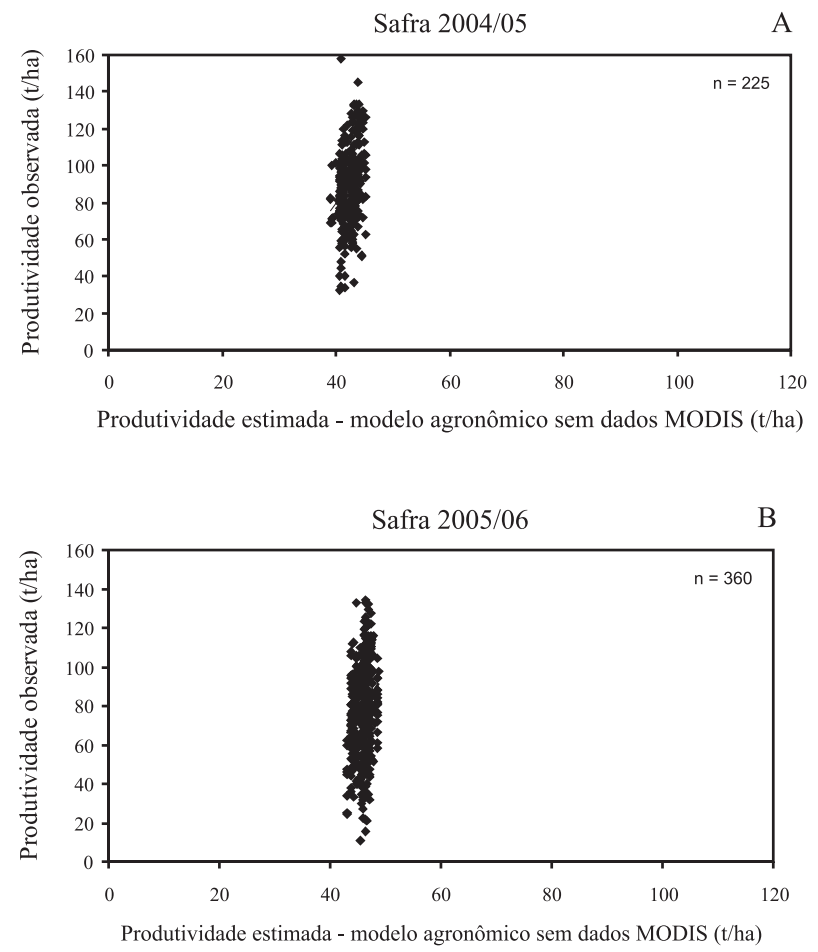

Figura 3. Relação entre a produtividade observada e a produtividade estimada pelo modelo agronômico utilizando o valor médio de NDVI por quinzena, nas safras (A) 2004/2005 e (B) 2005/2006.

\section{CONCLUSÃO}

Com o uso do modelo agronômico para estimativa da produtividade agrícola em talhões de cana verifica-se resultado promissor, principalmente devido à incorporação da variável espectral (índice de vegetação NDVI) no modelo, extraída das imagens do sensor MODIS, para cada talhão. Aparentemente, as variáveis meteorológicas não explicam qualquer variação da produtividade da cana entre talhões o que pode ser atribuído à sua baixa resolução espacial.

\section{AGRADECIMENTOS}

Aos proprietários da Usina Catanduva pelo fornecimento dos dados sobre os talhões de cana e ao 
engenheiro agrônomo Luiz Antonio Dias Paes, do Centro de Tecnologia Canavieira - CTC, pelo apoio na realização deste trabalho.

\section{REFERÊNCIAS}

BERKA, L.M.S.; RUDORFF, B.F.T.; SHIMABUKURO, Y.E. Soybean yield estimation by an agrometeorological model in a GIS. Scientia Agricola, v.60, p.433-440, 2003.

BLACK, T.L. The new NMC mesoscale Eta model: Description and forecast examples. Weather and Forecasting, v.9, p.265278, 1994.

CAMARGO, A.P. Balanço hídrico no Estado de São Paulo. Campinas: Instituto Agronômico, 1971. 24p. (Boletim 116)

CASTRO, P.R.C. Maturadores químicos em cana-de-açúcar. In: SEMANA DA CANA-DE-AÇÚCAR DE PIRACICABA, 4, 1999, Piracicaba. Anais... Piracicaba: Comissão Organizadora, 1999. p. 12-16.

CHOUDHURY, B.J.; AHMED N.U.; IDSO S.B.; REGINATO R.J.; DAUGHTRY C.S.T. Relations between evaporation coefficients and vegetation indices studied by model simulations. Remote Sensing of Environment, v.50, p.1-17, 1994.

DOORENBOS, J.; KASSAM, A.H. Yield response to water. Roma: Food and Agriculture Organization of the United Nations, 1979. 193p. (FAO - Irrigation and Drainage Paper n. 33)

FONTANA, D.C.; BERLATO, M.A.; LAUSCHNER, M.H.; MELO, R.W. Modelo de estimativa de rendimento de soja no Estado do Rio Grande do Sul. Pesquisa Agropecuária Brasileira, v. 36, p.399-403, 2001.

IBGE. Sistema IBGE de Recuperação Automática - SIDRA. Previsão de Safra. Cana-de-açúcar. Disponível em: http:/ / www.sidra.ibge.gov.br/. Acesso em março 2008.

LATORRE, M. L.; SHIMABUKURO, Y.E.; ANDERSON, L. O. Produtos para ecossistemas terrestres MODLAND. In: RUDORFF, B. F. T; SHIMABUKURO, Y. E.; CEBALLOS, J. C. (Coord.). Sensor MODIS e suas Aplicações Ambientais no Brasil. 1.ed. São José dos Campos: Editora Parêntese, 2007. p.23-36.

NORMAN, J.M.; ANDERSON, M.C.; KUSTAS, W.P.; FRENCH, A.N.; MECIKALSKI, J.; TORN, R.; DIAK, G.R.; ACHMUGGE, T.J. Remote Sensing of Evapotranspiration for Precision-Farming Applications. In: IEEE International Geoscience and Remote Sensing Symposium, 2003, Tolouse. Proceedings... Tolouse: IGARSS 2003 - Organizing Committee, 2003. p. 21-25.

PEREIRA, A.R.; VILA NOVA, N.A.; SEDIYAMA, G.C. Evapo(transpi)ração. Piracicaba: FEALQ, 1997. 183p.

RIZZI, R.; RUDORFF, B. F. T; FREITAS, R. M.; FONTANA, D. C. Monitoramento e estimativa da produtividade de soja auxiliados pelo modelo regional de previsão do tempo ETA. Ambiente e água: An Interdisciplinary Journal of Applied Science, v.1, p. 28-38, 2006.
RIZZI, R.; RUDORFF, B.F.T. Imagens do sensor MODIS associadas a um modelo para estimar a produtividade de soja. Pesquisa Agropecuária Brasileira, v. 42, p.73-80, 2007.

RUDORFF, B.F.T. Dados Landsat na estimativa da produtividade agrícola da cana-de-açúcar. 1985. 114p. Dissertação (Mestrado em Sensoriamento Remoto) - Instituto Nacional de Pesquisas Espaciais - INPE, São José dos Campos.

RUDORFF, B.F.T.; BATISTA, G.T. Yield estimation of sugarcane based on agrometeorological - Spectral models. Remote Sensing of Environment, v.33, p.183-192, 1990.

RUDORFF, B.F.T.; BATISTA, G.T. Wheat yield estimation at the farm level using Landsat-TM and agrometeorological data. International Journal of Remote Sensing, v. 2, p.2477-2484, 1991.

RUDORFF, B.F.T., SHIMABUKURO, Y.E., BATISTA, G.T., LEE, D. The contribution of qualitative variables to a sugarcane yield model based on spectral vegetation index. In: SIMPOSIO LATINOAMERICANO DE PERCEPCIÓN REMOTA, 7., 1995, Puerto Vallarta. Anais... Puerto Vallarta: Sociedad de Especialistas Latinoamericanos en Percepción Remota, 1995. p.705-708.

RUDORFF, B.F.T.; SHIMABUKURO, Y.E.; CEBALLOS, J.C. (Coord.). Sensor MODIS e suas Aplicações Ambientais no Brasil. 1.ed. São José dos Campos: Editora Parêntese, 2007. 425 p.

STEEL, R. G. D.; TORRIE, J. H. Principles and procedures of statistics: a biometrical approach. New York: McGraw-Hill, 1980. 633p.

TERAMOTO, E. R. Avaliação e aplicação de modelos de estimativa de produção de cana-de-açúcar (Saccharum spp) baseados em parâmetros do solo e do clima. 2003. 73p. Tese (Doutorado em Agronomia) - Universidade de São Paulo Escola Superior de Agricultura Luiz de Queiroz - ESALQ, Piracicaba.

WILLMOTT, C.J.; ACKLESON, S.G.; DAVIS, J.J.; FEDDEMA, K.M.; KLINK, D.R. Statistics for the evaluation and comparison of models. Journal of Geophysical Research, v.90, p.8995-9005, 1985. 